\title{
Effect of excess vacancy concentration on As and Sb doping in $\mathrm{Si}$
}

\author{
M Dalponte ${ }^{1}$, M C Adam ${ }^{1}$, H I Boudinov ${ }^{1}$, L V Goncharova ${ }^{2}$, T Feng ${ }^{3}$, \\ E Garfunkel $^{3}$ and T Gustafsson ${ }^{3}$ \\ ${ }^{1}$ Laboratorio de Mircoeletronica, Instituto de Fisica, Universidade Federal do Rio Grande do Sul, \\ 91501-970, Porto Alegre, RS, Brazil \\ ${ }^{2}$ Department of Physics and Astronomy, University of Western Ontario, London, Ontario, \\ Canada N6A 3K7 \\ ${ }^{3}$ Nanophysics Laboratory, Department of Physics and Astronomy, Rutgers University, \\ 136 Frelinghuysen Rd, Piscataway, NJ 08854, USA \\ E-mail: mateus.dalponte@ufrgs.br
}

Received 26 March 2009, in final form 22 June 2009

Published 31 July 2009

Online at stacks.iop.org/JPhysD/42/165106

\begin{abstract}
In defect engineering, defects are intentionally introduced in a substrate to modify dopant behaviour. In this work, we used high resolution depth profiling and electrical characterization to analyse $\mathrm{As}$ and $\mathrm{Sb}$ behaviour in Si substrates with and without excess vacancies. Vacancies were generated by nitrogen or oxygen ion pre-implantation prior to dopant (As or Sb) implantation. Separation by implanted oxygen (SIMOX) samples were also doped and analysed. The results obtained for Si without vacancies and SIMOX were similar in regard to dopant distribution, retention and their electrical characteristics. Vacancy-rich samples show different electrical characteristics and redistribution behaviour during annealing, attributed not just to the presence of vacancies but also to the ion used to create them. In the case of As doping, oxygen pre-implanted samples had a larger retained dose and less As interface accumulation, whereas for Sb doping, nitrogen pre-implanted samples presented a larger retained dose and less Sb interface accumulation. For both dopants, vacancy-rich samples had lower electrically active dopant concentrations, but better thermal stability of the activated dopants when compared with SIMOX and Si.
\end{abstract}

\section{Introduction}

As electronic devices reach higher performance and smaller power consumption, their decreasing dimensions require a better understanding of dopant behaviour during the various fabrication processes. Ion implantation is still widely used for introducing dopants into single-crystal substrates, despite the fact that this process introduces many crystal defects. It is therefore necessary to use a crystal recovery process, such as thermal annealing, to eliminate defects and to allow dopants to occupy substitutional positions in the lattice, so that they can become electrically active. This procedure is likely to remain the dominant method of MOSFET source/drain extension shallow junction formation in the near future [1]. In these junctions, highly doped, highly activated and low sheet resistance layers must be obtained [2,3]. The typical doping depth will be in the $10 \mathrm{~nm}$ range, which means that dopant diffusion during annealing and interaction with defects can have drastic effects on junction depth and hence on the device performance $[4,5]$.

Heavy elements, such as As and $\mathrm{Sb}$, are preferred n-type dopants for Si due to their low depth penetration during ion implantation, compared with the previously used phosphorous, and low diffusivity during thermal treatments. There were recently several reports showing that $\mathrm{Sb}$ is a strong candidate to replace As in future devices. Sb allows the formation of shallower junctions than As due to its larger mass and lower diffusion coefficient. However, its solid solubility (in the order of $4 \times 10^{19} \mathrm{~cm}^{-3}$ at $1000^{\circ} \mathrm{C}$ [6]) is approximately two orders of magnitude lower than that of As $\left(2 \times 10^{21} \mathrm{~cm}^{-3}\right.$ at $\left.1000^{\circ} \mathrm{C}[6]\right)$, resulting in a higher resistance of the doped layer. $\mathrm{Sb}$ is currently used in channel doping of metal-oxidesemiconductor field-effect-transistors for threshold voltage adjustment [1], where low concentrations are required. 
One possible method to modify dopant behaviour is the use of intentionally introduced substrate defects, for example vacancies. This technique has already been used successfully for boron $[7,8]$, reducing the diffusivity and increasing the level of electrical activation. Kong et al [9] showed that As diffusivity is also reduced by the presence of vacancies, but no electrical characterization was performed. Other works $[10,11]$ showed that $\mathrm{Sb}$ diffusivity is also strongly affected by the presence of vacancies. Opposed to B and As, vacancies caused an enhancement of Sb diffusivity, especially in the initial stage of the annealing, causing the well-known effect of transient enhanced diffusion (TED). The vacancies can be generated by ion implantation. The most commonly used ion is Si itself, but other species can be used as well [10-13]. In that case the dopant behaviour can be affected not only by the vacancies, but also by the implanted ion, through chemical interactions.

The purpose of this paper is to study the effect of vacancies created by pre-implantation of oxygen or nitrogen on two different n-type dopants, As and Sb, in Si. We also analysed $\mathrm{As}$ and $\mathrm{Sb}$ implantations in separation by implanted oxygen (SIMOX). SIMOX is one kind of silicon-on-insulator substrate which has received considerable attention $[14,15]$ due to its simplicity of fabrication and excellent top Si layer thickness uniformity. The buried oxide (BOX) is formed by a high dose of oxygen implantation. Since it is fabricated by oxygen implantation, the top Si layer presents higher oxygen concentration than bulk Si wafers. We checked for possible similarities between the behaviour of the dopants in SIMOX and in the samples with high vacancy concentration formed by oxygen implantation. Also, possible chemical effects related to the implantation of nitrogen were analysed. Besides ion scattering characterization, which was also performed in previous works $[16,17]$, electrical characterization, including doped layer sheet resistance, dopant electrical activation, mobility and charge carrier profiling, was also performed.

\section{Experimental}

(1 000 ) oriented SIMOX and p-type $\mathrm{Si}$ substrates with resistivities of $2-10 \Omega \mathrm{cm}$ were used. The native $\mathrm{SiO}_{2}$ layer was removed in diluted HF just before implantation. In the Si samples, a $150 \mathrm{~nm}$ deep vacancy-rich layer was formed by $\mathrm{O}^{+}$or $\mathrm{N}^{+}$ion implantation at $120 \mathrm{keV}$ using a dose of $5 \times 10^{16} \mathrm{~cm}^{-2}$ at $400{ }^{\circ} \mathrm{C}[12,13]$. From the results of de Souza et al $[12,13]$, the estimated vacancy concentration is on the order of $10^{19} \mathrm{~cm}^{-3}$. Following this pre-implantation, $\mathrm{As}^{+}$or $\mathrm{Sb}^{+}$was implanted at room temperature at $20 \mathrm{keV}$ to a dose of $5 \times 10^{14} \mathrm{~cm}^{-2}$. The low energy used guarantees that the As and $\mathrm{Sb}$ profiles are fully contained in the vacancy-rich layer (the peak concentration of both $\mathrm{As}$ and $\mathrm{Sb}$ in the as-implanted profile corresponds to a depth of less than $20 \mathrm{~nm}$ ). The arsenic, antimony, oxygen, nitrogen and vacancy profiles obtained from simulations with SRIM [18] are presented in figure 1.

The samples were then processed by rapid thermal annealing (RTA) at $1000{ }^{\circ} \mathrm{C}$ for $10 \mathrm{~s}$ or by furnace annealing (FA) at $1000{ }^{\circ} \mathrm{C}$ for $15 \mathrm{~min}$ in a nitrogen atmosphere. Similar As or Sb implantations were done in SIMOX and in a reference

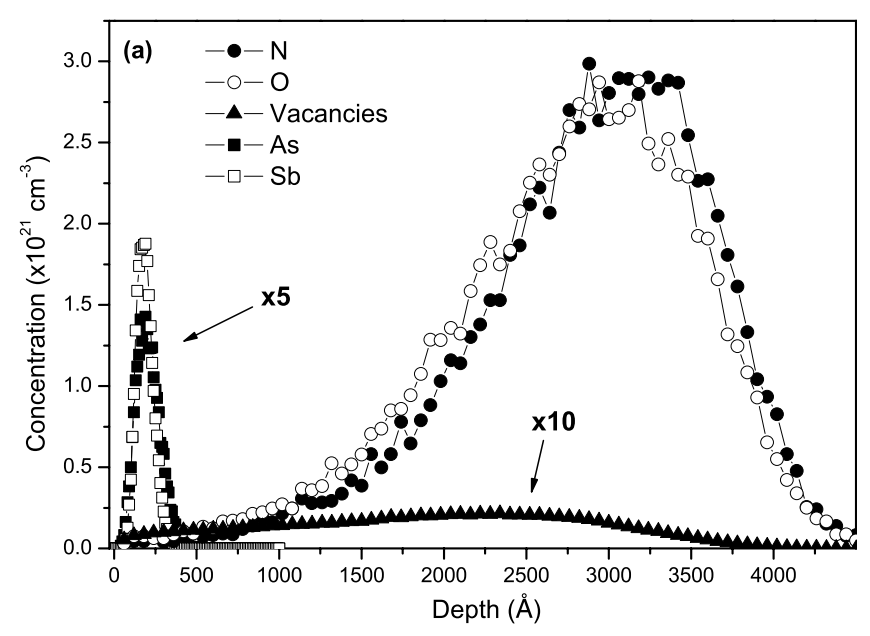

Figure 1. As, Sb, O, N and vacancy profiles obtained from SRIM. The vacancy concentration is estimated on the order of $10^{19} \mathrm{~cm}^{-3}$. The dopants are fully contained in the vacancy-rich layer.

Si sample without $\mathrm{N}$ or $\mathrm{O}$ pre-implantation. The samples were labelled based on the preparation scheme, shown in figure 2. The first part of the name refers to the implanted dopant, the second one to the substrate and the third one to the thermal treatment. The dopant labels were As or Sb; the substrate labels were $\mathrm{Si}$-for pure silicon without $\mathrm{N}$ or $\mathrm{O}$ pre-implantation (the reference sample); SIMOX-for SIMOX without $\mathrm{N}$ or $\mathrm{O}$ pre-implantation; $\mathrm{N}$ - for $\mathrm{Si}$ with $\mathrm{N}$ pre-implantation and $\mathrm{O}$-for $\mathrm{Si}$ with $\mathrm{O}$ pre-implantation. The thermal treatment labels were as-implanted (not annealed), RTA or FA. For example, As O RTA refers to a sample of silicon with oxygen pre-implantation, doped with As and then annealed in RTA.

Dopant concentration profiles were measured by medium energy ion scattering (MEIS) with a $\mathrm{H}^{+}$beam at an energy of $130.5 \mathrm{keV}$ in random and channelling alignments. MEIS is a high-resolution version of conventional Rutherford backscattering spectrometry (RBS), which allows quantitative depth profiling with near monolayer resolution in the near surface region. Backscattered ion energies were analysed with a high-resolution $(\Delta E / E \sim 0.1 \%)$ toroidal electrostatic energy detector resulting in a depth resolution of $\sim 0.9 \mathrm{~nm}$ at a depth of $50 \mathrm{~nm}$. The double alignment geometry was used in all channelling measurements to minimize the signal from the Si substrate. The beam was aligned in the $\left[\begin{array}{lll}1 & 0 & 0\end{array}\right]$ channelling direction and the detector position was centred on the [ $\left.\begin{array}{lll}1 & 1 & 1\end{array}\right]$ blocking direction, at a scattering angle of $125.5^{\circ}$. For the random geometry measurements, the sample was rotated in the sagittal plane in such a way that the incident beam was directed $9.5^{\circ}$ off the sample normal, but the backscattering angle remained constant. The surface approximation [19] with energy loss values extracted from the software SRIM [18] was used to convert the energy scale of the spectra into depth scale. A well-known relation between backscattering yield and concentration [19] was used to determine the dopant concentration profile. Using a similar procedure on the data measured in channelling alignment we obtained the interstitial dopants concentration profiles. Subtracting the interstitial profile (channelling) from the total profile (random) we obtain the substitutional dopants profile, which is compared 


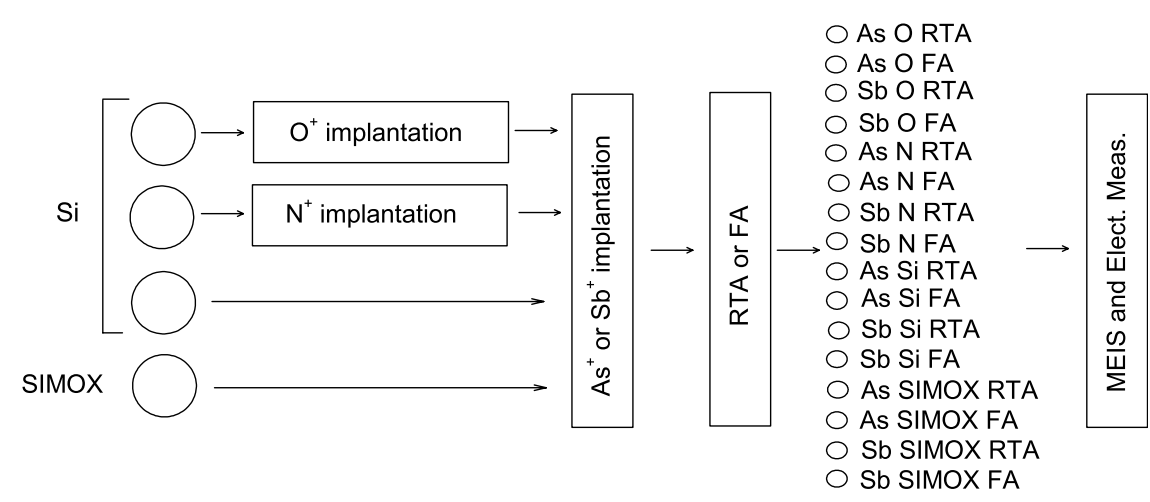

Figure 2. Sample preparation scheme with implantation, annealing and characterization steps, showing all prepared samples.

with the active dopant profile obtained by differential Hall measurements, as explained in the next paragraph.

Electrical characterization and Hall measurements were done on lithographically patterned samples with a Van der Pauw structure [20]. Sheet resistance $\left(R_{\mathrm{S}}\right)$, sheet carrier concentration $\left(n_{\mathrm{s}}\right)$ and carrier mobility $(\mu)$ were evaluated. The charge carrier concentration profiles (corresponding to the active dopant profiles) were extracted from differential Hall results. This technique consists of a repetition of electrical measurements (sheet resistance and Hall measurements) and thin surface layer removal steps. We have introduced the following method of Si etching, based on the model used by Bennet et al [21]. The incremental removal steps were done by oxidizing the sample surface in a $\mathrm{H}_{2} \mathrm{SO}_{4}: \mathrm{H}_{2} \mathrm{O}_{2}$ (4:1) solution at $120^{\circ} \mathrm{C}$ for $10 \mathrm{~s}$ and removing the oxide in a $\mathrm{HF}: \mathrm{H}_{2} \mathrm{O}$ DI $(1: 8)$ solution also for $10 \mathrm{~s}$. These oxidation and oxide removal steps are repeated five times between each electrical measurement. The etching steps were performed until the measured $n_{\mathrm{s}}$ reached a stable value that represented a negligible fraction of the $n_{\mathrm{s}}$ measured before the first etching step. The total Si thickness removed between each electrical measurement is $25 \AA$, which was calibrated by RBS on SIMOX samples doped with As or Sb and etched by a different number of steps. The calibration was done with SIMOX because the thickness of the top Si layer can be easily determined by RBS after the etch steps, and thus, the removed thickness can be calculated. We assumed that the same etching rate occurred in bulk Si samples as well. In every electrical measurement we obtained $R_{\mathrm{s}}, n_{\mathrm{s}}$ and $\mu$. Using the relations $[6,22]$

$$
\begin{aligned}
\frac{1}{R_{\mathrm{s}, i}} & =\frac{1}{R_{\mathrm{s}, i+1}}+\frac{1}{R_{\mathrm{s}, \text { layer }}}, \\
n_{\mathrm{s}, i} & =n_{\mathrm{s}, i+1}+n_{\mathrm{s}, \text { layer }}, \\
\mu_{\text {layer }} & =\frac{1}{e \times n_{\mathrm{s}, \text { layer }} \times R_{\mathrm{s}, \text { layer }}}
\end{aligned}
$$

we obtained the values of $R_{\mathrm{s} \text {,layer }}, n_{\mathrm{s} \text {, layer }}$ and $\mu_{\text {layer }}$ for each removed layer, where $R_{\mathrm{s}, i}, R_{\mathrm{S}, i+1}$ and $R_{\mathrm{s} \text {, layer }}$ are the sheet resistances before the removal step, after the removal step and of the removed layer, respectively; $n_{\mathrm{s}, i}, n_{\mathrm{s}, i+1}$ and $n_{\mathrm{s}, \text { layer }}$ are the sheet carrier concentrations of the sample before the removal step, after the removal step and of the removed layer; $\mu_{\text {layer }}$ is mobility of the charge carriers in the removed layer and $e$ is the electron charge. Dividing $n_{\mathrm{s} \text {, layer }}$ by the layer thickness ( $25 \AA$ ) we obtained the charge carrier concentration in each layer and thus the charge carrier concentration profile.

\section{Results and discussion}

\subsection{Retained and interface-accumulated dopant concentrations}

Figure 3 shows the profiles of As after (a) RTA and (b) FA extracted from MEIS spectra measured in a random direction and compared with their respective as-implanted profiles. Figure 4 shows the Sb profiles. The as-implanted profiles of As and $\mathrm{Sb}$ are very similar to each other, but differ strongly in the annealed samples due to their different diffusivities. The retained doses, the accumulated dopant doses at the $\mathrm{SiO}_{2} / \mathrm{Si}$ interface and the non-accumulated doses (the difference between the retained and the accumulated doses, i.e. the dose retained far from the interface) were calculated from the profiles and are presented in table 1 as a percentage of the implanted dose. The retained dose was calculated as the integral over the whole dopant concentration profile. The accumulated dose was estimated as the integral of part of the profile, from the surface to a depth of approximately $75 \AA$, i.e. the peak that appears at the interface with the native oxide.

In the case of As (figure 3), there was a considerable diffusion in all samples even after short annealing times (RTA), especially in the As N RTA sample. Nitrogen is known to trap vacancies [23], reducing the amount of available vacancies for As, thus allowing it to diffuse faster than in the samples where the vacancies were not trapped, causing the largest dopant loss and accumulation at the interface with the surface $\mathrm{SiO}_{2}$ (see figure 3(a) and table 1). In the As O RTA samples, on the other hand, vacancies act as nucleation centres for $\mathrm{SiO}_{2}$ precipitates by forming complexes with oxygen [24]. Possibly the formation of these precipitates reduce As diffusivity, since $\mathrm{SiO}_{2}$ is a barrier for As diffusion. This effect was not as significant as the effect of nitrogen, as the retained and accumulated doses in the $\mathrm{O}$ sample were just slightly larger than in the $\mathrm{Si}$ and SIMOX samples, indicating that oxygen does not trap vacancies so efficiently as nitrogen. After FA, the excess vacancies have recombined, making the As profiles at depths larger than $75 \AA$ very similar in all samples. 


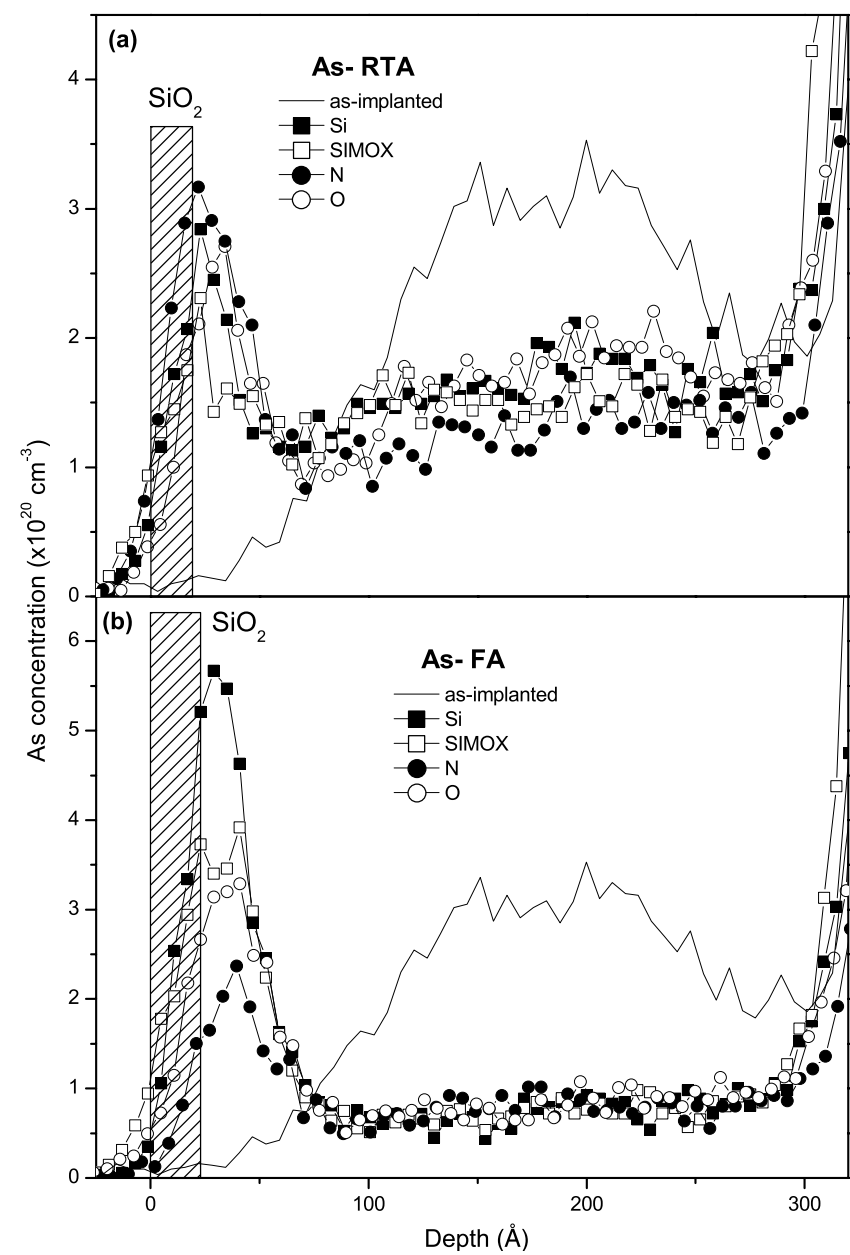

Figure 3. As profiles after ( $a$ ) RTA and (b) FA. After RTA, the As profiles showed considerable diffusion and accumulation at the interface. After FA, the amount of As accumulated at the interface increased, except in the As N RTA, where an unexpected reduction was observed.

The main differences were observed in the amount of As accumulated at the interface with the surface $\mathrm{SiO}_{2}$. In the nitrogen pre-implanted sample this amount decreased when compared with the RTA sample, in contrast to what happened in all the other samples. Considering the non-accumulated dose, we note that it is the same in the Si, SIMOX and N samples and is larger in the $\mathrm{O}$ sample. This means that the As loss in the $\mathrm{N}$ sample originated mainly from the As accumulated in the interface. In the low energy region of the MEIS spectrum of the As N FA sample (not shown), nitrogen was detected at the interface with the $\mathrm{SiO}_{2}$. The presence of nitrogen in that region of the sample prevented As from accumulating there. This might happen due to the competition between As and $\mathrm{N}$ for vacancies and available sites at the interface. With fewer vacancies and lattice sites available for As, its diffusivity is not reduced allowing it to out-diffuse more easily than in the samples where the vacancies are not trapped.

Nitrogen was detected at the interface only in the FA sample and not in the RTA sample because in the latter case the thermal budget was not enough to allow nitrogen to diffuse all the way up to the interface, since it was implanted at a depth of more than $2000 \AA$. The same effect was observed for

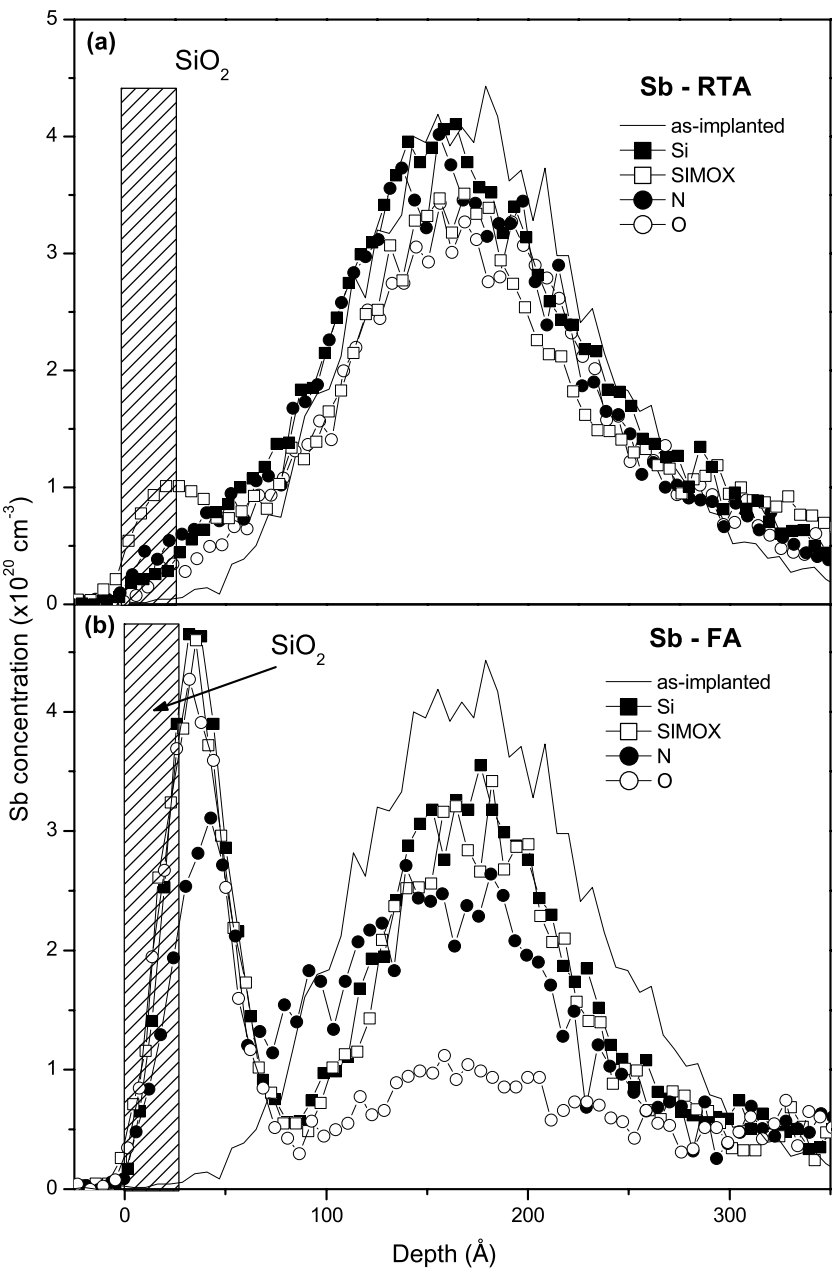

Figure 4. Sb profiles after (a) RTA and (b) FA. After RTA, Sb remains practically immobile. After FA, all samples presented increase in the amount of $\mathrm{Sb}$ accumulated at the interface with surface $\mathrm{SiO}_{2}$.

oxygen in the oxygen pre-implanted samples. In the As O FA sample MEIS spectrum (not shown) we observed an increase in the surface oxide thickness (a long tail of the low energy edge of the oxygen peak, typical of sub-stoichiometric oxides) related to the diffusion of the pre-implanted oxygen up to the interface. It caused lower accumulation of As than in the $\mathrm{Si}$ and SIMOX samples, although larger than in the $\mathrm{N}$ sample, but still reducing the number of available vacancies for As. In the O sample, the oxygen that reached the interface contributed to the increase in the surface oxide thickness, which acts as a diffusion barrier for As. In this case, the oxidation of the interface prevents the accumulation of As not by facilitating its out-diffusion, as in the case of nitrogen, but by retaining it in the bulk, as can be seen by the non-accumulated dose, which was the largest of all.

From the MEIS point of view, the vacancy-rich ( $\mathrm{N}$ and O pre-implanted) samples presented very distinct behaviour, evidencing that As diffusion was strongly influenced by the pre-implanted ion. The oxygen pre-implanted samples had the highest retained doses of all samples after both RTA and FA, although the difference to the Si and SIMOX samples was small. They also showed an amount of accumulated As equal 
Table 1. Retained, accumulated at the interface with the surface oxide, and non-accumulated doses after thermal treatments in all samples. Doses were calculated from dopant concentration profiles. The percentage of the total implanted dose of $5 \times 10^{14} \mathrm{~cm}^{-2}$ is shown. The error is less than $1.5 \%$ of the implanted dose.

\begin{tabular}{|c|c|c|c|c|c|}
\hline Dopant & Annealing & Substrate & $\begin{array}{l}\text { Retained } \\
\text { dose } \\
\text { (\% of impl. dose) }\end{array}$ & $\begin{array}{l}\text { Accumulated } \\
\text { dose } \\
\text { (\% of impl. dose) }\end{array}$ & $\begin{array}{l}\text { Non-accumulated } \\
\text { dose } \\
\text { (\% of impl. dose) }\end{array}$ \\
\hline \multirow[t]{8}{*}{ As } & RTA & $\mathrm{Si}$ & 94 & 20 & 74 \\
\hline & & SIMOX & 92 & 20 & 72 \\
\hline & & $\mathrm{N}$ & 78 & 24 & 54 \\
\hline & & $\mathrm{O}$ & 96 & 20 & 76 \\
\hline & FA & $\mathrm{Si}$ & 70 & 38 & 32 \\
\hline & & SIMOX & 64 & 32 & 32 \\
\hline & & $\mathrm{N}$ & 48 & 16 & 32 \\
\hline & & $\mathrm{O}$ & 72 & 26 & 46 \\
\hline \multirow[t]{8}{*}{$\mathrm{Sb}$} & RTA & $\mathrm{Si}$ & 100 & 6 & 94 \\
\hline & & SIMOX & 94 & 10 & 84 \\
\hline & & $\mathrm{N}$ & 100 & 8 & 92 \\
\hline & & $\mathrm{O}$ & 88 & 6 & 82 \\
\hline & FA & $\mathrm{Si}$ & 82 & 28 & 54 \\
\hline & & SIMOX & 94 & 28 & 66 \\
\hline & & $\mathrm{N}$ & 84 & 20 & 64 \\
\hline & & $\mathrm{O}$ & 58 & 26 & 32 \\
\hline
\end{tabular}

to or lower than in the Si and SIMOX samples. Conversely, the nitrogen pre-implanted samples showed the lowest retained doses and the highest accumulated dose, in the case of RTA. In the case of FA, the accumulated dose was the lowest, but it is related to the highest dopant loss. Apparently, oxygen acted as an As diffusion reducer by forming $\mathrm{SiO}_{2}$ precipitates, while nitrogen enhanced As diffusion by trapping vacancies that normally bind to As and slow its diffusivity. As our results indicate, the major role in dopant distribution was played by the interactions between the pre-implanted ions and the vacancies and not by the interaction of the vacancies with As, since oxygen and nitrogen pre-implanted samples had similar vacancy concentrations and presented very distinct behaviour after annealing. Further studies are necessary to confirm these hypotheses.

A comparison between figures 3 and 4 shows that the $\mathrm{Sb}$ implanted samples presented little diffusion after RTA, compared with As. Most of the $\mathrm{Sb}$ atoms remained in their as-implanted distribution and the retained doses were high. Unlike As, the lowest retained dose was seen in the oxygen pre-implanted sample, not in the nitrogen one. Sb diffuses exclusively via vacancies and the trapping of vacancies by nitrogen reduces its diffusivity resulting in a high retained dose. The ability of oxygen to trap vacancies was, as observed with As, not as efficient as nitrogen's, allowing Sb out-diffusion and causing the largest loss. After FA (figure 4(b)), the shape of the profile remained basically unchanged, except for some reduction in concentration and the appearance of a peak of accumulated dopants at the interface with the surface oxide. The retained doses decreased, but not considerably. With $\mathrm{Sb}$, the accumulated dose at the interface after FA was larger than after RTA for all samples without exceptions. Also, the total accumulated dose of $\mathrm{Sb}$ was, in most cases, smaller than that of As, due to the smaller diffusivity of $\mathrm{Sb}$.
An interesting fact is that for $\mathrm{Sb}$ the nitrogen pre-implanted samples presented better results than the oxygen pre-implanted ones, opposite to what was observed with As. For Sb, the nitrogen pre-implanted samples had higher retained doses than the oxygen pre-implanted ones for both RTA and FA. In terms of the accumulated dose, the Sb N RTA had a larger dose than the Sb O RTA, but the difference was small and after FA, the situation is reversed, i.e. the Sb O FA sample had a much higher amount of accumulated dopants than the $\mathrm{Sb} \mathrm{N}$ FA sample, especially if we consider the ratio of accumulated dopants to the retained dose. We believe that the more efficient vacancy trapping by nitrogen than by oxygen caused a slower diffusion of $\mathrm{Sb}$ in the $\mathrm{N}$ sample. Sb behaviour in the $\mathrm{N}$ sample was similar or slightly better than in the samples without excess vacancies, $\mathrm{Si}$ and SIMOX. this shows that the number of available vacancies for $\mathrm{Sb}$ diffusion in these three samples was practically similar, i.e. the excess vacancy concentration was quickly trapped by nitrogen.

\subsection{Substitutionality and electrical activation of the dopants}

An important characteristic for doping is the substitutional dose, i.e. the number of dopants in substitutional sites per $\mathrm{cm}^{2}$. Figure 5 shows selected profiles extracted from random and channelling alignment MEIS spectra of $(a)$ As and $(b) \mathrm{Sb}$. The most noticeable difference is the much higher Sb interstitial concentration compared with As. In terms of the dopants accumulated at the interface, in the case of $\mathrm{Sb}$, the peaks from random and channelling spectra always coincide, indicating that the dopants at the interface are not substitutional. In the case of As, there is always a small fraction of the accumulated dopants that are substitutional. The As OFA sample is the most interesting case, where, despite an increase in accumulated dose when compared with the As O RTA sample, a large part of 


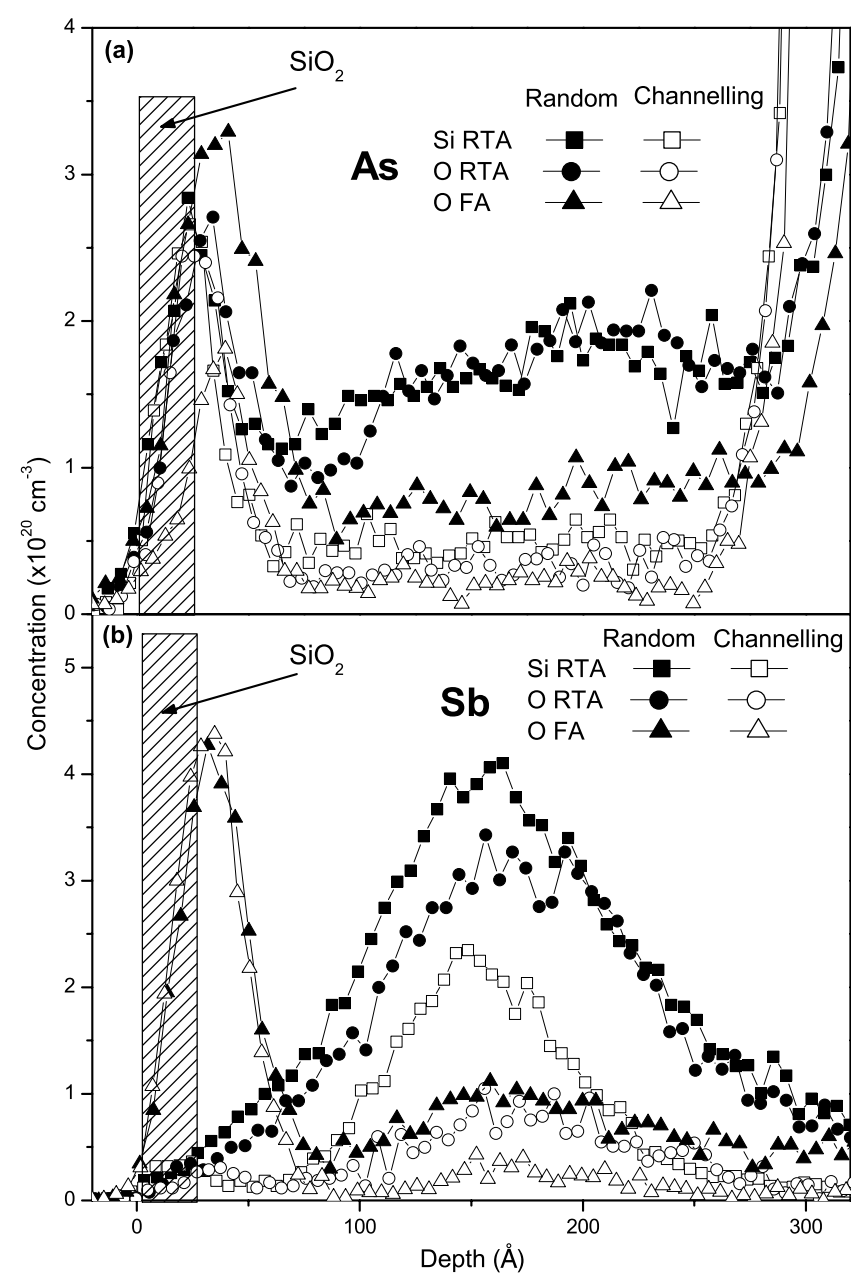

Figure 5. Total (random) and interstitial (channelling) profiles of (a) As and (b) Sb. The substitutionality at depths beneath $75 \AA$ is excellent in $(a)$ and part of the As accumulated at the interface is also substitutional, especially in the As O FA sample. In $(b)$ a large fraction of $\mathrm{Sb}$ is in interstitial sites, especially in the samples after RTA.

the accumulated dopants were substitutional (see figure $5(a)$ ). Again, for As, the oxygen pre-implanted samples presented better results than the nitrogen pre-implanted ones (see table 2): the substitutional doses were the highest in both As O RTA and As O FA. However, for Sb, the advantages presented by the nitrogen pre-implanted samples in terms of retained dose previously discussed did not occur in terms of the substitutional dose. The substitutional doses were larger in the Sb N RTA and Sb N FA than in the Sb O RTA and Sb O FA, respectively, but not significantly.

Analysing the data in table 2, we note that the electrically active dose of As is larger than the substitutional dose in the same sample, in almost every sample except As O RTA. This implies that part of the dopants that were visible to the proton beam used in MEIS analysis, mainly those accumulated at the interface with $\mathrm{SiO}_{2}$ (supposedly interstitials), are electrically active. This effect is related to the poor channelling of the proton beam in the near interface region. After passing through the amorphous surface oxide, the beam reaches the interface and needs to travel a few Angstrom in the crystalline substrate before it becomes 'channelled'. This causes the near interface substitutional dopants to be detected as interstitials. The accumulated As peak at the interface seems to be interstitial, but its deep region (around $50 \AA$, in figure $5(a)$ ) presents some level of channelling, indicating that the beam is starting to channel in that region. Nevertheless, the As located closer to the interface appears completely interstitial, since the beam is not channelled yet, so it is not possible to differentiate between substitutional and interstitial dopants. The large electrically active As dose indicates that those dopants cannot be interstitial, since in this case they would not be active. Hence we believe that the high interstitial As dose at the interface is just an artefact of MEIS, leading to an underestimation of the substitutional dose and to a discrepancy between substitutional and electrically active doses. For Sb, this effect can be neglected because, as can be seen in figure 5(b), the accumulated $\mathrm{Sb}$ peak in random and channelling alignments coincide as a whole, i.e. there is no channelling even in the deep region where the beam is already channelled, indicating that the dopants are actually completely interstitial.

The Si and SIMOX samples presented the expected results, i.e. the activation is higher after RTA than after $\mathrm{FA}$, due to the deactivation of $\mathrm{As}$ and $\mathrm{Sb}$ as a function of increasing annealing times [25-27]. The sheet resistance and carrier mobility values increased, which is in agreement with a reduction in activation. In the $\mathrm{N}$ and $\mathrm{O}$ pre-implanted samples different behaviour was observed. The active doses remained approximately stable after FA when compared with RTA. The carrier mobility increased most likely due to the recovery of implantation damage. As a consequence of the stability of the active dose and the increase in mobility, the sheet resistance values decreased by reasonable amounts, which is unexpected for As and $\mathrm{Sb}$ [28], since the common behaviour is the dopant deactivation accompanied by an increase in sheet resistance as observed in Si and SIMOX. Despite the interesting reduction in sheet resistance, the measured values were not significantly better than those measured in the $\mathrm{Si}$ and SIMOX samples for the treatments used in this work. This is because after a long thermal treatment the excess vacancies have recombined and the dopants' electrical activation is expected to be similar to the samples without excess vacancies.

\subsection{Differential Hall measurements}

The nitrogen pre-implanted samples presented large values of active dopant doses, but this effect was related to the activation of nitrogen, which is a column $\mathrm{V}$ element, like As and Sb, which makes it an n-type dopant, despite being a poor dopant, with extremely low activation percentages. By the differential Hall technique, the nitrogen's contribution to the active dose could be discounted from the measured values. The final results are those presented in table 2 . The active doses are similar in the nitrogen and oxygen pre-implanted samples. This indicates that the vacancies play the main role in activation (or better stated, in deactivation), without any chemical effects of the pre-implanted ion on the dopants.

Figure 6 shows active dopant profiles obtained by differential Hall measurements for $(a)$ As and $(b) \mathrm{Sb}$ as well as substitutional dopant profiles of selected samples, calculated 
Table 2. Substitutional and electrically active doses, sheet resistance and charge carriers mobility of all samples after thermal treatments. The percentage of the total implanted dose of $5 \times 10^{14} \mathrm{~cm}^{-2}$ is shown. The errors are indicated on the head of each column.

\begin{tabular}{|c|c|c|c|c|c|c|}
\hline Dopant & Annealing & Substrate & $\begin{array}{l}\text { Substitutional } \\
\text { dose (MEIS) } \\
(\% \text { of impl. dose }) \pm 1 \%\end{array}$ & $\begin{array}{l}\text { Active dose, } n_{\mathrm{s}} \\
(\% \text { of impl. dose }) \\
\pm 1.75 \%\end{array}$ & $\begin{array}{l}R_{\mathrm{s}} \\
\left(\Omega \mathrm{sq}^{-1}\right) \\
\pm 0.5 \%\end{array}$ & $\begin{array}{l}\text { Mobility } \\
\left(\mathrm{cm}^{2} \mathrm{~V}^{-1} \mathrm{~s}^{-1}\right) \\
\pm 1.75 \%\end{array}$ \\
\hline \multirow[t]{8}{*}{ As } & \multirow{4}{*}{ RTA } & $\mathrm{Si}$ & $74 \%$ & $85 \%$ & 221 & 67 \\
\hline & & SIMOX & $76 \%$ & $89 \%$ & 219 & 64 \\
\hline & & $\mathrm{N}$ & $64 \%$ & $75 \%$ & 259 & 64 \\
\hline & & $\mathrm{O}$ & $80 \%$ & $73 \%$ & 383 & 44 \\
\hline & \multirow[t]{4}{*}{ FA } & $\mathrm{Si}$ & $34 \%$ & $69 \%$ & 231 & 78 \\
\hline & & SIMOX & $36 \%$ & $65 \%$ & 233 & 83 \\
\hline & & $\mathrm{N}$ & $34 \%$ & $70 \%$ & 196 & 90 \\
\hline & & $\mathrm{O}$ & $58 \%$ & $68 \%$ & 224 & 81 \\
\hline \multirow[t]{8}{*}{$\mathrm{Sb}$} & \multirow[t]{4}{*}{ RTA } & $\mathrm{Si}$ & $74 \%$ & $74 \%$ & 230 & 73 \\
\hline & & SIMOX & $68 \%$ & $66 \%$ & 253 & 74 \\
\hline & & $\mathrm{N}$ & $72 \%$ & $32 \%$ & 479 & 82 \\
\hline & & $\mathrm{O}$ & $68 \%$ & $32 \%$ & 554 & 70 \\
\hline & \multirow{4}{*}{ FA } & $\mathrm{Si}$ & $34 \%$ & $32 \%$ & 386 & 100 \\
\hline & & SIMOX & $30 \%$ & $29 \%$ & 419 & 104 \\
\hline & & $\mathrm{N}$ & $34 \%$ & $25 \%$ & 476 & 107 \\
\hline & & $\mathrm{O}$ & $32 \%$ & $31 \%$ & 404 & 99 \\
\hline
\end{tabular}

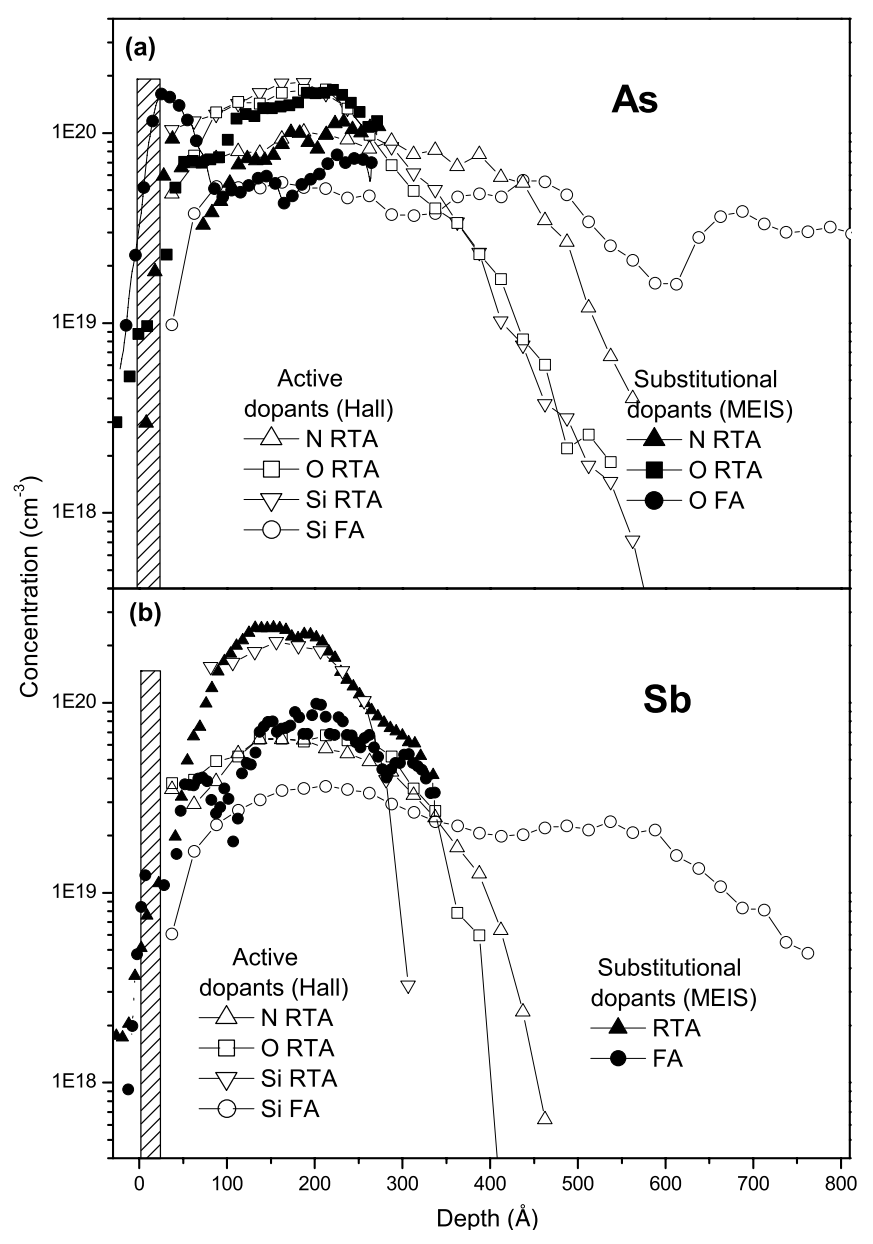

Figure 6. Substitutional and active dopants profiles for $(a)$ As and (b) Sb extracted from MEIS and differential Hall measurements, respectively (selected samples). In $(a)$ the profiles coincide, showing that the substitutional As is electrically active in all samples, while in $(b)$ part of the substitutional $\mathrm{Sb}$ is not active in the vacancy-rich samples. from MEIS profiles. The substitutional profiles are truncated at a certain depth due to the different geometries used in random and channelling MEIS analysis. The different geometries cause the superposition of the Si substrate signal of the random measurement to the deep part of the dopant profile obtained from the channelling measurement. When the subtraction of the channelling from the random spectra is performed to obtain the substitutional profiles, part of the substitutional dopant profiles is left out (the part superimposed by the substrate signal), decreasing the substitutional dose. This effect is noticed in both As and $\mathrm{Sb}$ (as can be seen by the truncated substitutional profiles in figures $6(a)$ and $(b))$, but it is more relevant for $\mathrm{Sb}$, since the effect related to the accumulated peak, as previously explained, occurs only for As. The truncation leads to an additional underestimation of the substitutional dose. The substitutional dopants profiles can only be integrated to a certain depth that does not comprise the whole profile. Hence, the substitutional dose presented in table 2 is actually lower than the real substitutional dose, especially in the FA samples, where the profiles are deeper and the truncation causes a larger part of the profiles to be left out of the integration. In figure 6(a) substitutional and active As profiles are shown. After RTA, the substitutional profiles in the As Si RTA, As SIMOX RTA and As O RTA were similar, thus only the As O RTA sample is shown. Also the active dopant profiles in these samples show similar shapes, despite concentration differences. As mentioned earlier (see figure 3( $a$ ) and table 1), the diffusion of As was more pronounced in the As N RTA sample, which can also be observed in figure 6(a), where the As in this sample presents a broader profile with lower peak concentration. After FA, all substitutional profiles were similar, except for the peak of substitutional As accumulated at the interface with the surface oxide in the As O FA sample that was not observed in the other samples. For this reason only the profile in this sample is shown. Regarding the active 
dopant profile, they were all similar, hence only the As Si FA is shown. This also applies to the As O FA sample, where the substitutional As accumulated at the interface was not electrically active. Again the active and substitutional dopant profiles were in good agreement. All profiles presented similar concentrations and shapes, indicating that after long thermal treatments neither vacancies (already recombined) nor pre-implanted ions influenced electrical activation.

Figure 6(b) shows the Sb profiles which showed a different behaviour from that of As. First, the substitutional Sb profiles were similar in all samples after each annealing, RTA or FA, (hence they are named RTA and FA in the figure, regardless of the kind of substrate). However, large differences were observed in the active $\mathrm{Sb}$ profiles after RTA: in the $\mathrm{Si}$ and SIMOX samples they presented similar concentrations as in the substitutional profiles, but a shallower distribution. In the $\mathrm{N}$ and $\mathrm{O}$ samples they presented a much lower concentration, evidencing that a large fraction of substitutional $\mathrm{Sb}$ is not electrically active. The profiles were deeper than in the $\mathrm{Si}$ and SIMOX samples, showing larger diffusivity. Both effects are likely to be associated with the interaction with vacancies. After FA, the active $\mathrm{Sb}$ profiles were similar in all samples, thus only the profile in Sb Si FA is shown. A small fraction of substitutional $\mathrm{Sb}$ was electrically inactive in all samples, as can be seen in the lower concentration of the active Sb profiles when compared with the substitutional ones. Likewise observed for As, after long thermal treatments neither vacancies nor pre-implanted ions influenced electrical activation.

The active dopant concentration profiles in the nitrogen pre-implanted samples for both $\mathrm{As}$ and $\mathrm{Sb}$ were always similar or lower than the profiles in the corresponding oxygen pre-implanted sample (see figure 6). Also, the sheet carrier concentration always presented a similar value as in the corresponding oxygen pre-implanted samples. The dopant active dose was similar in the nitrogen and oxygen pre-implanted samples, i.e. the pre-implanted ions had no effect on both dopants' electrical activation, which was affected only by vacancies, mainly after the short annealing, when the vacancies had not recombined yet.

\section{Conclusions}

Complementary ion scattering measurements and electrical characterization were applied to understand the behaviour of $\mathrm{As}$ and $\mathrm{Sb}$ in different substrates (single crystal $\mathrm{Si}$, SIMOX and two types of vacancy-rich Si: nitrogen or oxygen pre-implanted) and the effects of excess vacancies. Analysing the dopants redistribution, clear differences were observed between the samples with and without vacancies. We observed a dependence on the pre-implanted ion. For As dopants, the oxygen pre-implanted samples presented larger retained and substitutional doses after both RTA and FA compared with the nitrogen pre-implanted samples. The nitrogen pre-implanted samples presented the smallest retained and substitutional As doses. For Sb, oxygen and nitrogen had an opposite trend than that observed for As, i.e. the nitrogen pre-implanted samples were better with larger retained and substitutional $\mathrm{Sb}$ doses. We believe that it is due to the interaction of the pre-implanted ions and the dopants with vacancies. Nitrogen easily traps vacancies allowing As to diffuse faster and reducing $\mathrm{Sb}$ diffusivity. Oxygen uses vacancies as $\mathrm{SiO}_{2}$ precipitates nucleation centres, but this process seems to be slower than the trapping by nitrogen. We infer this based on the fact that As diffusion was slower in the oxygen pre-implanted samples, typical of As-vacancy complexes, and that Sb diffusion was faster in these samples, typical behaviour of $\mathrm{Sb}$ associated with vacancies.

Regardless of the pre-implanted ion, the most important differences in dopant electrical characteristics were due to the presence or not of vacancies. The vacancy-rich samples presented similar dopant activation after both thermal treatments and for both dopants. The results were inferior to those presented by the samples without excess vacancies, $\mathrm{Si}$ and SIMOX. One advantage presented by the vacancy-rich samples was the thermal stability of the active dopants after long annealing times. While the Si and SIMOX samples presented the expected dopant deactivation, the $\mathrm{N}$ and $\mathrm{O}$ samples presented similar activation values after both thermal treatments. In the latter samples, the combination of the stable activation values with the increase in carrier mobility after FA compared with RTA resulted in a reduction in sheet resistance, which is not a typical behaviour for As and Sb.

The active dopants profiles differ for $\mathrm{As}$ and $\mathrm{Sb}$. In the case of As, they basically followed the substitutional dopant profiles. In the case of $\mathrm{Sb}$, the active dopant and substitutional profiles coincided only in the Si and SIMOX RTA samples. In the other samples, the active dopant profiles were always lower than the substitutional ones, especially in the vacancy-rich samples annealed in RTA, where a large amount of substitutional dopant was not active. After FA, the substitutional profiles decreased and became more similar to the active dopants profiles, but still higher.

In summary, for the thermal treatments used in this work, the dopants' electrical activation was controlled by the excess vacancies which caused dopant deactivation independent of the pre-implanted ion. The dopants' redistribution (retained and accumulated doses) was controlled by the pre-implanted ion, most likely through the interaction with vacancies, but, nevertheless, nitrogen and oxygen played the most important roles, displaying opposite effects for As and Sb.

\section{Acknowledgments}

This work was supported in part by the National Science Foundation DMR 0706326, by the Semiconductor Research Corporation, USA, and by the Brazilian agencies CAPES and CNPq.

\section{References}

[1] Semiconductor Industry Assoc. International SEMATECH 2005 The International Technology Roadmap for Semiconductors (Austin, TX)

[2] Kim S-D, Park C-M and Woo J C S 2002 IEEE Trans. Electron Devices 491748

[3] Uedono A, Chen Z Q, Ogura A, Suzuki R, Ohdaira T and Mikado T 2002 J. Appl. Phys. 916488 
[4] Jones C and Ishida E 1998 Mater. Sci. Eng. R 241

[5] Chen Z Q, Uedono A, Ogura A, Ono H, Suzuki R, Ohdaira T and Mikado T 2002 Appl. Surf. Sci. 194112

[6] Jaeger R C 1988 Introduction to Microelectronics Fabrication, Modular Series on Solid State Devices vol 5 (Reading, MA: Addison-Wesley)

[7] Shao L, Zhang J, Chen J, Tang D, Thompson P E, Patel S, Wang X, Chen H, Liu J and Chu W-K 2004 Appl. Phys. Lett. 843325

[8] Smith A J, Colombeau B, Gwilliam R, Cowern N E B, Sealy B J, Milosavljevic M, Collart E, Gennaro S, Bersani M and Barozzi M 2005 Mater. Sci. Eng. B 124-125 210

[9] Kong N, Banerjee S K, Kirichenko T A, Anderson S G H and Foisy M C 2007 Appl. Phys. Lett. 90062107

[10] Lulli G, Bianconi M, Solmi S, Napolitani E and Carnera A 2000 J. Appl. Phys. 878461

[11] Dey S and Varma S 2001 J. Appl. Phys. 902618

[12] de Souza J P, Suprun-Belevich Yu, Boudinov H and Cima C A 2000 J. Appl. Phys. 878385

[13] de Souza J P, Suprun-Belevich Yu, Boudinov H and Cima C A 2001 J. Appl. Phys. 8942

[14] Colinge J P 1997 SOI Technology: Materials to VLSI (Dordrecht: Kluwer)

[15] Cristoloveanu S and Sheng S L 1995 Electrical Characterization of Silicon-On-Insulator Materials and Devices (Dordrecht: Kluwer )

[16] Dalponte M, Boudinov H, Goncharova L V, Garfunkel E and Gustafsson T 2006 AIP Conf. Proc. 931137
[17] Dalponte M, Boudinov H, Goncharova L V, Garfunkel E and Gustafsson T 2006 Nucl. Instrum. Methods Phys. Res. B 249874

[18] Ziegler J F and Biersack J P 2003 SRIM: The Stopping and Range of Ions in Matter, version 2003.26, available at http://www.srim.org

[19] Chu W-K 1978 Backscattering Spectrometry (New York: Academic)

[20] Van der Pauw L J 1958/59 Philips Tech. Rev. 20220

[21] Bennet N S, Smith A J, Colombeau B, Gwilliam R, Cowern N E B and Sealy B J 2005 Mater. Sci. Eng. B 124-125 305-309

[22] Blood P and Orton J W 1992 The Electrical Characterization of Semiconductors: Majority Carriers and Electron States (San Diego: Academic)

[23] Voronkov V V and Falster R 2004 Mater. Sci. Eng. B 114-115 130-4

[24] Voronkov V V and Falster R 2003 Mater. Sci. Semicond. Process. 5 387-90

[25] Pandey K C, Erbil A, Cargill G S, Boheme R F and Vanderbilt D 1998 Phys. Rev. Lett. 6111

[26] Rousseau P M, Griffin P B, Fang W T and Plummer J D 1998 J. Appl. Phys. 847

[27] Takamura Y, Vailionis A, Marshall A F, Griffin P B and Plummer J D 2002 J. Appl. Phys. 925503

[28] Takamura Y, Jain S H, Griffin P B and Plummer J D 2002 J. Appl. Phys. 92 230-234 